Journal of Anesthesia

123 words in abstract

3637 words excluding abstract, acknowledgment, refs \& legends

3 Figures 64 Refs

\title{
Nociceptin system as a target in sepsis?
}

Róisín Thomas, Cordula Stover, David G. Lambert and Jonathan Thompson*

University Department of Cardiovascular Sciences, Division of Anaesthesia, Critical Care and Pain Management, University of Leicester, Leicester Royal Infirmary, Leicester, United Kingdom, LE1 5WW, UK.

*Address correspondence and reprint requests to Dr Jonathan $\mathrm{P}$ Thompson at the above address.

Tel: +44 (0)116 252 3132. email: jt23@leicester.ac.uk 


\begin{abstract}
The nociceptin system comprises the nociceptin receptor (NOP) and the ligand which binds to the receptor (N/OFQ). The archetypal role of the system is in pain processing but it is also expressed on immune cells. Activation of the NOP receptor is known to modulate inflammatory responses such as mast cell degranulation, neutrophil rolling, vasodilation, increased vascular permeability, regulation of adhesion molecules and recruitment of leucocytes. As there is a loss of regulation of inflammatory responses during sepsis, the nociceptin system could be a target for therapies aimed at modulating sepsis. This review details the known effects of NOP activation on leucocytes and the vascular endothelium and discusses the most recent human and animal data on the nociceptin system in sepsis.
\end{abstract}

Short Title: The nociceptin system and sepsis

Key Words: Nociceptin, N/OFQ, ppNoc, NOP, Sepsis. 


\section{Introduction}

The inflammatory response involves activation of several cell types in blood and tissues to initiate a cascade of events to remove pathogens and repair damaged tissue. Sepsis occurs when dysregulation of the inflammatory response occurs and localised inflammation becomes systemic (often referred to as a cytokine storm). This is accompanied by a compensatory anti-inflammatory response, which may lead to a state of immune paralysis ${ }^{1}$. The pathogenesis of sepsis involves a series of interacting neuro-endocrine immune and inflammatory pathways which cause circulatory and cellular dysfunction (with impaired mitochondrial function and oxygen utilisation). These have widespread effects which can result in multi-organ failure. The reported annual incidence of severe sepsis is $50-90$ per 100,000 population, ${ }^{2}$ and the incidence of sepsis is 3-4 times higher than this. Severe sepsis is increasing in incidence because of an ageing population with chronic morbidities, increasing medical interventions, and the development of antimicrobial resistance. ${ }^{23}$ Mortality from severe sepsis is estimated at $28-50 \%$, with recent overall hospital mortality rates of $36 \%$ from European and $39.8 \%$ from UK-ICUs respectively. ${ }^{4}$ Sepsis affects all age groups and with incidence increasing, mortality morbidity and economic costs of treatment are all set to rise.

Despite advances in understanding the mechanisms and pathways involved, global interventions (e.g. to improve oxygen delivery) or specific interventions targeted at components of these pathways (e.g. TNF antagonists, anti-endotoxin antibodies) have been unsuccessful. Possible reasons are:

1. Sepsis is a clinical syndrome comprising a biological response to a heterogeneous variety of insults rather than a distinct disease entity.

2. Exaggerated responses to inflammatory cytokines or other pathways may be adaptive and not necessarily harmful per se.

3. Interventions aimed at specific downstream mediators of sepsis may be limited when upstream mechanisms are not addressed.

4. Inflammatory or immune responses vary between individuals and over the clinical course of sepsis. Initially exaggerated inflammatory responses are often followed by depression of aspects of immune function.

5. Inflammatory responses have significant genetic variability.

6. Extrapolations of data from animal or laboratory models may be limited and translational research is needed.

Therefore, further research is required to understand the pathogenesis and define the differences between responses to infective and non-infective responses. Because of innate variability in 
responses it is logical to target endogenous systems, in particular responses early after an inflammatory stimulus (upstream).

One such endogenous system that has been implicated in modulation of the immune response in sepsis is the nociceptin/orphanin FQ (N/OFQ) system. ${ }^{5,6}$

The nociceptin system comprises the nociceptin receptor (NOP) and the ligand that binds to the receptor (N/OFQ). The ligand is a peptide of 17 amino acids in length and is defined as a non-classical member of the opioid family, since it has a similar sequence to classical opioids but it does not bind to their receptors. ${ }^{6} \mathrm{~N} / \mathrm{OFQ}$ has many other roles distinct from its role in the pain pathway (for which it is most well-known), such as roles in depression, anxiety, stroke and heart failure. ${ }^{8}$

\section{Leucocytes, cytokines and the N/OFQ system in sepsis.}

The first indication that the nociceptin system may be involved with regulation of immune responses arose in 1995 when NOP mRNA was detected on mouse lymphocytes, human lymphocytes and lymphocytic cell lines. ${ }^{6}$ Subsequently, NOP mRNA was found in the thymus, lymph nodes, spleen and splenocytes of pigs ${ }^{9}$ and more types of human leucocytes (Figure 1). mRNA for prepronociceptin (ppN/OFQ), the precursor for nociceptin, was later also found to be expressed on human peripheral blood lymphocytes. ${ }^{10}$ Further evidence of the involvement of the Nociceptin system has arisen from studies examining the effects of activating or antagonising the NOP receptor, or the effects changes in the system during conditions of inflammation or sepsis. Miller and Fulford showed that inflammatory stimuli can cause N/OFQ release in splenocytes. They incubated a crude splenocyte culture with $25 \mu \mathrm{g} / \mathrm{ml}$, lipopolysaccharide (LPS) a component of the cell wall in gram negative bacteria that is responsible for initiating an immune response, and measured N/OFQ concentrations at regular intervals over a period of $48 \mathrm{~h}$. They found that N/OFQ concentrations rose at around $18 \mathrm{~h}$ post stimulation. ${ }^{11}$

An increased peripheral leucocyte count is usually found as part of the inflammatory response in patients with sepsis. ${ }^{12}$ Data have shown that N/OFQ has a modulatory role in the chemo-attraction of leucocytes by mast cells. ${ }^{13}$ Mast cells release histamine, which is also increased in sepsis ${ }^{14}$ and affects vascular permeability ${ }^{15}$ and upregulates adhesion molecules ${ }^{16}$ (which also show upregulation in sepsis ${ }^{17}$ ). Kimura et al. found that intradermal injection of N/OFQ causes histamine release from rat peritoneal mast cells. This response could be inhibited by both calcium and pertussis toxin but not by naloxone, which provides evidence that this was due to NOP receptor activation. These 
authors also found that N/OFQ increased vascular permeability in rats in a dose dependent manner, which could be prevented using a $\mathrm{H} 1$ histamine receptor antagonist. ${ }^{13}$

The concept that N/OFQ can affect the vasculature by stimulating histamine release from mast cells was supported later by work performed by our group. Using fluorescent intravital microscopy, we found that N/OFQ-induced macromolecular leak could be inhibited by $\mathrm{H} 1$ and $\mathrm{H} 2$ receptor antagonists or by UFP-101. ${ }^{18}$ UFP-101 is a peptide antagonis that is selective for the NOP receptor. ${ }^{19}$ We also found that N/OFQ elicited other inflammatory responses such as vasodilation (also inhibited by the above antagonists) and increased rolling and adhesion of leucocytes on the endothelium ${ }^{18}$. More recently we used fluorescent-labelled N/OFQ to demonstrate that NOP receptors are expressed on rat mesenteric arterioles and venules. ${ }^{20}$

There is also evidence to suggest that N/OFQ may have the ability to recruit neutrophils directly. Serhan et al. found that functional NOP receptors are expressed on human neutrophils and performed a micro-chamber assay with isolated human neutrophils, which demonstrated that that N/OFQ is a potent chemoattractant forthese cells. ${ }^{21}$

In addition, N/OFQ may aid the infiltration of leucocytes via increasing endothelial expression of cellular adhesion molecules. This in turn can increase the interactions between endothelial cells and leucocytes and hence increase the numbers of leucocytes infiltrating inflamed tissues. ${ }^{22}$ Kato et al. found that the number of neutrophils, lymphocytes and macrophages present in the colonic mucosa of dextran sulphate sodium-treated mice (a model of colitis) was lower in NOP-knockout mice suggesting NOP is involved in the recruitment of all of these cell types; there was also significantly higher expression of mucosal vascular adhesion molecule 1 (MAdCAM-1) in wild type mice compared to NOP deficient mice. ${ }^{22}$ This suggests that N/OFQ is involved in the upregulation of adhesion molecules such as MAdCAM-1, which binds to lymphocytes and eosinophils) Evidence from our group shows that N/OFQ also upregulates the b2-integrin CD18/CD11b adhesion molecule on rat neutrophils. ${ }^{18}$ Increased interactions of neutrophils and other leucocytes within the microvasculature could lead to vascular occlusion. ${ }^{23,}{ }^{24}$ Therefore, one of the mechanisms by which N/OFQ could be contributing to the hypoperfusion and hypoxia that occurs in sepsis could be via upregulation of adhesion molecules. Unpublished data from our group shows that NOP is expressed on eosinophils. The significance of this is not yet known but these cells could also be contributing in a similar manner to that described for neutrophils.

N/OFQ may also be involved in triggering leucocyte effector functions. Trombella et al. found that N/OFQ promotes the release of lysozyme from neutrophils. ${ }^{25}$ Lysozyme is an enzyme that hydrolyses 
glycosidic bonds in the cell wall of some micro-organisms thereby killing them. Brown et al. suggest that the organ dysfunction that occurs in sepsis could be a result of excessive activation of neutrophils. ${ }^{26}$ This could represent a mechanism whereby N/OFQ contributes to decreased plasma circulating volume. N/OFQ (which is also expressed by neutrophils in patients with sepsis ${ }^{27}$ ) and the inflammatory cytokine, tumour necrosis factor alpha (TNF- $\alpha$ ) could be working together to cause neutrophil activation; this could then lead to vascular damage and increased vascular permeability. A hypothetical mechanism illustrating this concept is outlined in Figure 2.

TNF- $\alpha$ contributes to many of the pathophysiological processes involved in sepsis by initiating a cascade of events leading to inflammatory mediator release and upregulation of adhesion molecules on endothelial cells. ${ }^{28}$ It has also been found that apoptosis of neutrophils is delayed in patients with sepsis $^{26,29}$ which may be due to high concentrations of TNF- $\alpha$ found during sepsis. TNF- $\alpha$ is known to inhibit neutrophil apoptosis in vitro. ${ }^{30}$ and also to hinder migration of neutrophils from the circulation into inflamed tissues. ${ }^{31}$

When neutrophils are not active they are in either a resting or a primed state. Priming occurs when a resting neutrophil encounters microbial-derived or pro-inflammatory molecules. These encounters then switch the resting neutrophil from an inactive state to a state of readiness. Further encounters with the molecules that led to the initial priming cause neutrophils to become active, resulting in responses such as degranulation. Degranulation should happen in the tissues rather than the vasculature, however, oxidative activity and enhanced expression of the transcription factor NFKB, have been found in the circulating neutrophils of patients with sepsis, ${ }^{32-34}$ indicating that there is an increase in primed neutrophils during sepsis. This could be related to the increase in N/OFQ that has been observed in the plasma of patients who died from sepsis, compared with patients who survived. ${ }^{35}$

Neutrophil numbers rapidly rise during acute inflammation and peak after around 12 hours. ${ }^{36}$ As the inflammatory reaction progresses the recruitment of neutrophils is downregulated; neutrophils begin to apoptose and monocyte recruitment is upregulated. Monocytes are produced in the bone marrow and circulate in the blood before migrating to tissues where they differentiate into macrophages and dendritic cells and continue the eradication of pathogens but also contribute to the resolution of inflammation. ${ }^{37}$

Peluso et $a I^{38}$ showed that monocytes express mRNA for NOP receptors and Trombella et al. ${ }^{25}$ found that N/OFQ is a potent chemoattractant for these cells. This is significant because patients with sepsis have an increased monocyte count in their plasma ${ }^{39}$ to which N/OFQ could be 
contributing. Monocytes perform tasks such as antigen presentation and phagocytosis of pathogens and apoptotic cells, and macrophage activation is believed to play a central role in mediating sepsis. $^{40}$

Another important function of monocytes is production of pro-inflammatory cytokines, such as TNF$\alpha$ and Interleukin-1 beta (IL-1 $\beta$ ) both of which have been shown to affect mRNA concentrations and secretion of N/OFQ. TNF- $\alpha$, and IL-1 $\beta$ exposure causes N/OFQ mRNA concentrations to increase in rat astrocytes ${ }^{41}$ and incubation of unstimulated rat splenocytes with IL-1 $\beta$ increases N/OFQ secretion. ${ }^{11}$ The effects of TNF- $\alpha$ on neutrophils have been discussed previously but TNF- $\alpha$ has other pro-inflammatory actions such as upregulation of the expression of intracellular adhesion molecules that are important for leucocyte extravasation, such as ICAM-1 and VCAM-1 ${ }^{26}$ on endothelial cells. IL$1 \beta$ also upregulates adhesion molecules and induces chemokines, ${ }^{42}$ so recruitment of monocytes by N/OFQ leads to an increase in proinflammatory cytokines. The relationship between N/OFQ and proinflammatory cytokines was demonstrated by Goldfarb et al. who showed that TNF- $\alpha$ mRNA concentrations in the spleen and plasma increase significantly if N/OFQ is given before staphylococcal enterotoxin A challenge (a method of inducing inflammation). However, it had previously been shown that there was a reduced expression of TNF- $\alpha$ and IL-1 $\beta$ in peritoneal macrophages when N/OFQ was administered intracerebroventricularly to rats. ${ }^{43}$ Therefore, the route of administration (and thus, the source of endogenous N/OFQ) seems to be important in the immune responses observed previously. Goldfarb et al. also found that concentrations of the proinflammatory cytokine IFN- $\gamma$ which activates (and is secreted by) monocytes, also rose in the spleen in response to N/OFQ. These responses were diminished with ppN/OFQ knockouts. ${ }^{44}$

Activated monocytes also produce chemokines such as CCL2/MCP-1 (chemotactic for monocytes, T cells and dendritic cells) and CCL5/RANTES (a chemoattractant for T cells, eosinophils, and basophils). Carvalho et al. found increased CCL2/MCP-1 in septic rats that had been given N/OFQ ${ }^{45}$. However, Kaminsky and Rogers investigated the effect of N/OFQ on expression of CCL2/MCP-1 and also CCL5/RANTES in vitro by monocytes and found that production of CCL2/MCP-1 is supressed in human monocytes. ${ }^{46}$ This again highlights the complexity of the role of N/OFQ in modulating the immune system. N/OFQ can neither be seen as having anti-inflammatory nor pro-inflammatory effects on the immune system and is often referred to in the literature as having a modulatory effect. $^{47}$

In terms of the development of sepsis, the anti-inflammatory effects of NOFQ could be just as important as the pro-inflammatory effects. Impaired lymphocyte responsiveness and decreased 
lymphocyte numbers are observed in sepsis ${ }^{48}$ and N/OFQ has been shown to suppress IL-2 (a cytokine important for T-cell proliferation and differentiation) production and inhibit lymphocyte proliferation. ${ }^{11}$ Lymphocyte suppression results in a decrease in proinflammatory cytokines, and chemokines and therefore reduces infiltration and activation of leucocytes. Other anti-inflammatory actions associated with N/OFQ include the inhibition of the release of the pro-inflammatory

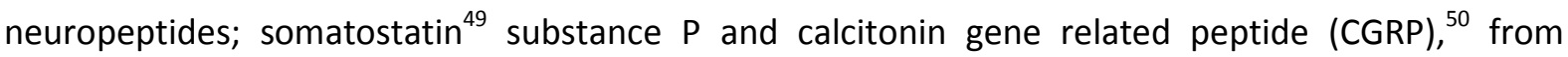
sensory nerve terminals.

A recent study further illustrates that the effects of N/OFQ on immune function are complex, showing that different doses can produce opposite effects: Petrella et al. administered N/OFQ by intraperitoneal injection to rats, after inducing colitis by infusing trinitrobenzenesulfonic acid into the colon. ${ }^{51}$ At lower doses, $(0.02$ and $0.2 \mathrm{nmol} / \mathrm{kg})$ improvements in microscopic damage and myeloperoxidase (indicative of neutrophil infiltration) activity, along with decreased IL-1 $\beta$ concentrations, were observed. Conversely, a high dose $(20 \mathrm{nmol} / \mathrm{kg})$ resulted in a worsening of colitis. Hence the in-vivo effects of N/OFQ seem to be determined by both the source, and the dose. Whilst colitis is not directly related to sepsis, these findings demonstrate the involvement of N/OFQ in immune modulation.

\section{Cardiovascular effects of Nociceptin}

Sepsis has profound effects on the cardiovascular system and there is substantial evidence that N/OFQ modulates cardiovascular responses. When given peripherally, intravenous N/OFQ has been shown to result in hypotension and bradycardia in rats $^{52}$ guinea pigs ${ }^{53}$ and mice. ${ }^{54}$ NOP is expressed in the human brain stem ${ }^{55}$ and hypothalamus ${ }^{52}$ (both important in cardiovascular regulation) and N/OFQ has been found to be expressed in human brain stem. ${ }^{55}$ Hypotension and bradycardia also occur in response to intracerebroventricular administration of $\mathrm{N} / \mathrm{OFQ}^{8,52}$ responses that can be abolished by antagonising the NOP receptor with UFP-101. ${ }^{6,57}$ Administration of N/OFQ via microinjection directly into the rostral ventrolateral medulla and paraventricular nucleus (areas of the brain also involved in cardiovascular regulation) also reduces blood pressure and heart rate compared to injection of the same volume of saline. ${ }^{58}$ These responses are not seen in NOP knockout animals. ${ }^{57}$

\section{Studies of the nociceptin system during sepsis in animal models}

Several studies have examined the role of the nociceptin system in animal models of inflammation and sepsis. For example, Carvalho et al. used a caecal ligation and puncture (CLP) model of sepsis to investigate the effects, on the inflammatory response, of activating, or antagonizing NOP with a 
range of doses of either N/OFQ or UFP-101 ${ }^{45}$. CLP involves ligation and puncture of the cecum in order to create fecal peritonitis. Rats were either killed after 12 hours in order to carry out biochemical analysis or observed to measure differences in mortality over a period of ten days.

Mortality in rats treated with UFP-101 in doses of 0.03 and $0.3 \mathrm{mg} / \mathrm{kg}$ was significantly reduced compared to rats that had undergone CLP and received no treatment (50\% and $70 \%$ mortality respectively), whereas mortality at 5 days was $100 \%$ in rats treated with of N/OFQ $0.1 \mathrm{mg} / \mathrm{kg}$ alone. The reduction in mortality with UFP-101 treatment occurred in the first two days after CLP, and the authors proposed that the nociceptin system is involved in the early phases of the inflammatory response in sepsis ${ }^{45}$. In order to assess recruitment of inflammatory cells, fluid was collected from peritoneal and bronchoalveolar lavage and cells were counted. Blood and peritoneal exudates were examined for differences in bacterial count and finally, blood cytokine and chemokine concentrations (TNF- $\alpha$, IL-1 $\beta$, IL-10 and MCP-1) were measured by ELISA Carvalho et al confirmed that leucocyte migration was significantly increased in septic rats compared to controls and that this could either be increased further by treatment with N/OFQ or inhibited by UFP-101. UFP-101 also substantially reduced bacterial spread. Treatment with N/OFQ led to increased plasma concentrations of CCL2/MCP-1, TNF- $\alpha$ and IL-1 $\beta$ whilst UFP-101 decreased plasma concentrations of these cytokines (in comparison to septic rats). These results led the authors to conclude that antagonising NOP could be useful in the treatment of sepsis by preventing both infiltration and bacterial spread.

In another CLP model of sepsis, Laufenberg et al. found that ppN/OFQ mRNA is increased in the stellate ganglion neurons of rats during sepsis and that the potency of N/OFQ appeared to be increased after 72 hours. ${ }^{592}$ Using whole cell patch clamping these investigators found that the dose of N/OFQ required to inhibit the NOP-mediated, voltage gated calcium current by $50 \%$ decreased from $78 \mathrm{nM}$ in non-septic rats to $60 \mathrm{nM}$ in septic rats. This is significant because it suggests that the nociceptin system could contribute to decreased neurotransmission in these neurons during sepsis and thus decreased sympathetic innervation of the heart. Therefore, NOP antagonists have the potential to increase neurotransmission in the stellate ganglion and hence improve cardiac function during sepsis.

A further study in our laboratories demonstrated that vasodilation induced by N/OFQ is enhanced after administration of LPS in rats. ${ }^{20}$ Furthermore, we showed that antagonising NOP with UFP-101 during LPS-induced sepsis produced anti-inflammatory effects (such as decreased macromolecular leak and reduced leucocyte rolling) in post-capillary venules. 


\section{Studies of the nociceptin system during sepsis in humans}

Data from rodents can only be used to inform clinical research, and studies in humans are clearly more relevant. We conducted a study involving patients with sepsis who had been admitted to the Intensive Care Unit (ICU) and found that patients who subsequently died had higher plasma concentrations of N/OFQ than those who survived, ${ }^{37}$. However, this was a small study and further data are needed.

Another study in humans was performed on whole blood samples from patients with sepsis in ICU. Stamer et al. found significantly higher expression of NOP receptor mRNA in ICU patients, and also in patients with cancer compared to healthy controls. ${ }^{60}$ However, ppN/OFQ mRNA expression was lower than in controls. This could be due to more ppN/OFQ being cleaved into N/OFQ. In another study, Zhang et al. stimulated peripheral whole blood samples from healthy volunteers with LPS, TNF- $\alpha$, IL-1B, IL-10 or IFN- $\gamma$ and then measured concentrations of NOP and ppN/OFQ mRNA in leucocytes and cytokine concentrations in the supernatant. They found that NOP was suppressed by LPS, TNF- $\alpha$, IL-1 $\beta$, IL-10 and IFN- $\gamma$ whilst ppN/OFQ was suppressed only by LPS and IL-10 (which is an anti-inflammatory cytokine), suggesting the involvement of negative feedback mechanisms. ${ }^{61}$ The same group also sought to elucidate the mechanism of the changes in the expression of NOP and ppN/OFQ under inflammatory conditions. This was investigated using anti-cytokine antibodies to determine whether the suppression of NOP and ppN/OFQ concentrations were affected if individual cytokines were removed. LPS-induced NOP suppression was partially alleviated by incubation with either anti-TNF- $\alpha$ or anti-IL-1 $\beta$ antibodies, indicating that these cytokines are involved in the regulation of NOP. A potential mechanism could be that NOP induces TNF- $\alpha$ and IL-1 $\beta$ and they then feedback to prevent further expression of NOP and thus further release of TNF- $\alpha$ and IL-1B. However antibodies to TNF- $\alpha$ and IL-1 $\beta$ only partially prevented the suppression of NOP and made no difference to concentrations after 6 hours so the mechanisms of regulation of the system is clearly more complex and relies on more than just these two cytokines. Moreover, ppN/OFQ concentrations were not affected by the anti-cytokine antibodies at all, indicating that ppN/OFQ is regulated in a different way.

Our group recently carried out a study of the effect of sepsis on the nociceptin system. ${ }^{78}$ We assessed the relationship between the nociceptin system and clinical outcomes of patients who were either admitted to intensive care with sepsis or who represented a model of systemic inflammation (patients undergoing cardiac bypass, which induces a similar pro inflammatory 
cytokine release profile as that observed in sepsis). The relationship between pro and antiinflammatory cytokines was also investigated. Concentrations of ppNOFQ and NOP mRNA were measured by qPCR in isolated polymorphonuclear leukocytes, a radioimmunoassay was used to determine concentrations of N/OFQ in plasma and TNF- $\alpha$, IL- 8 and IL-10 in plasma were measured by ELISA.

Patients with sepsis showed an increase in plasma N/OFQ concentrations and both pro and antiinflammatory cytokines compared to controls whereas concentrations of ppN/OFQ and NOP mRNA in polymorphs decreased. A similar but lesser effect was observed in cardiac bypass patients confirming that there is upregulation in the nociceptin system in a non-septic model of inflammation. The finding that ppNOFQ mRNA is decreased in sepsis is in agreement with the previously mentioned findings of Stamer et al. However, unlike their group we did not find differences between patients with or without a diagnosis of cancer and we found a decrease as opposed to their increase in NOP mRNA expression in sepsis. Some of the discrepancies between these studies may be related to methodological differences, including the use of whole blood or polymorphonuclear cells isolated from blood samples for $\mathrm{qPCR}$, and the measurement of N/OFQ protein rather than mRNA alone.

In this recent study we also found that cytokine concentrations were higher in non-survivors of sepsis $^{27}$; however, in contrast to our earlier study we did not find any relationship between plasma concentrations of N/OFQ and patient survival. This illustrates the heterogeneity of clinical studies of sepsis in humans, the limitations of current clinical diagnostic criteria for sepsis and the potential effects of co-existing disease or clinical decisions regarding withdrawal of treatment on mortality in clinical practice. Our findings that there were significant increases in inflammatory cytokines linked to increased plasma N/OFQ concentrations do, however, indicate that there may be a link with upregulation of the nociceptin system and increased morbidity. Further studies will be necessary to attempt to address the question of whether this is related to an increase in deaths from sepsis.

Taken together the data from the studies discussed above suggest that upregulation of the nociceptin system may be detrimental to health whereas a decrease could be advantageous (Figure $3)$.

\section{Summary}


Modulation of the immune system by the nociceptin system is extremely complex and the details are not fully elucidated. Further are studies are needed to better understand the effects of activating or antagonising the system and to explain the conflicting results in terms of up or downregulation of the system during inflammation and sepsis. However, the available data indicate that a substantial number of processes are affected by the nociceptin system in inflammation and sepsis; sepsis has a high mortality and there may be substantial benefits in therapeutic modulation of the nociceptin system. 


\section{Expression of NOP on Leucocytes}

dunovivg $\quad$ Expresses NOP
$? \quad$ Unknown if this cell expresses NOP
?

Haematopoetic Stem Cell I

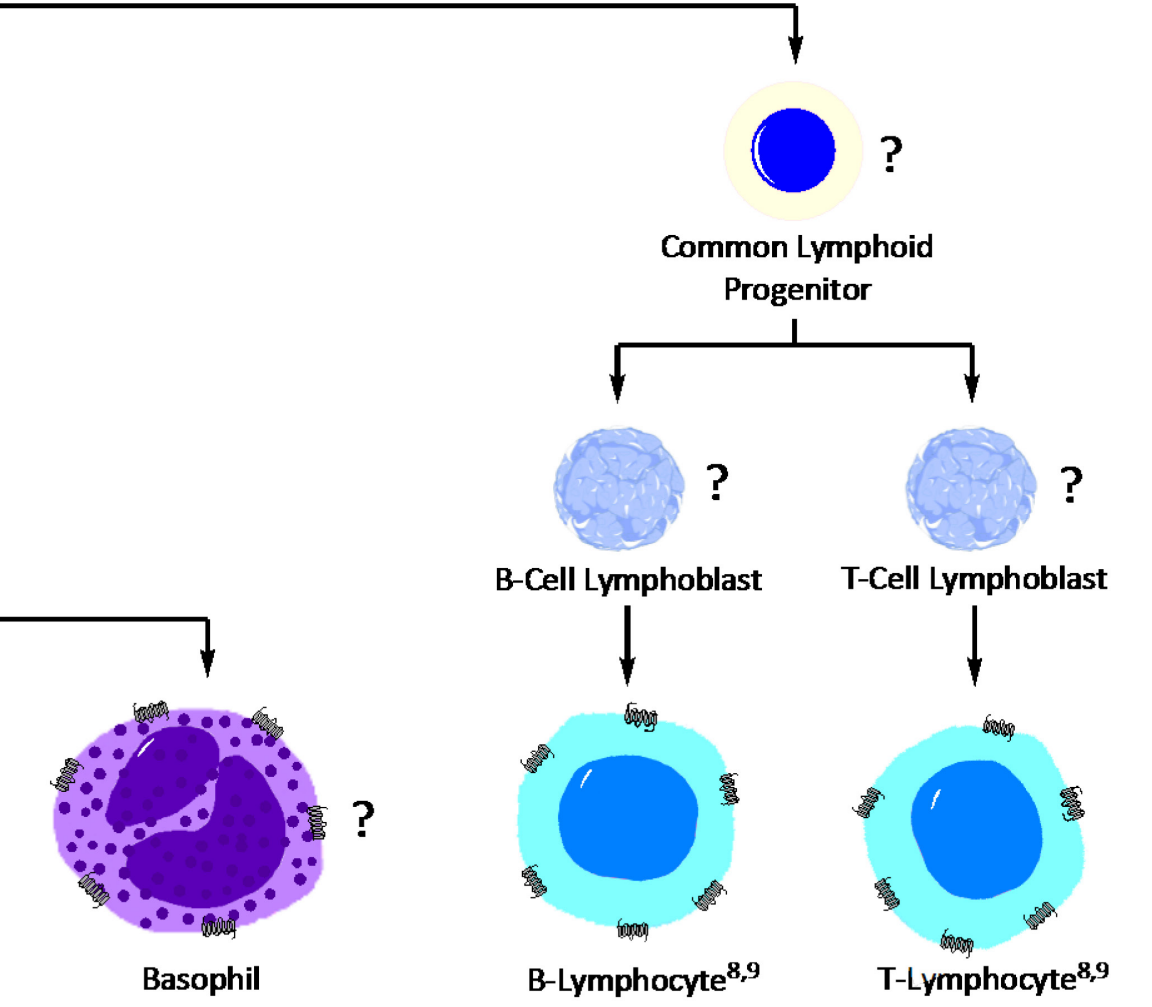

Figure 1 NOP expression on white blood cells. NOP is expressed on all mature leucocytes. * Unpublished data from our laboratory. 


\section{Possible mechanism for N/OFQ induced vascular permeability in sepsis}

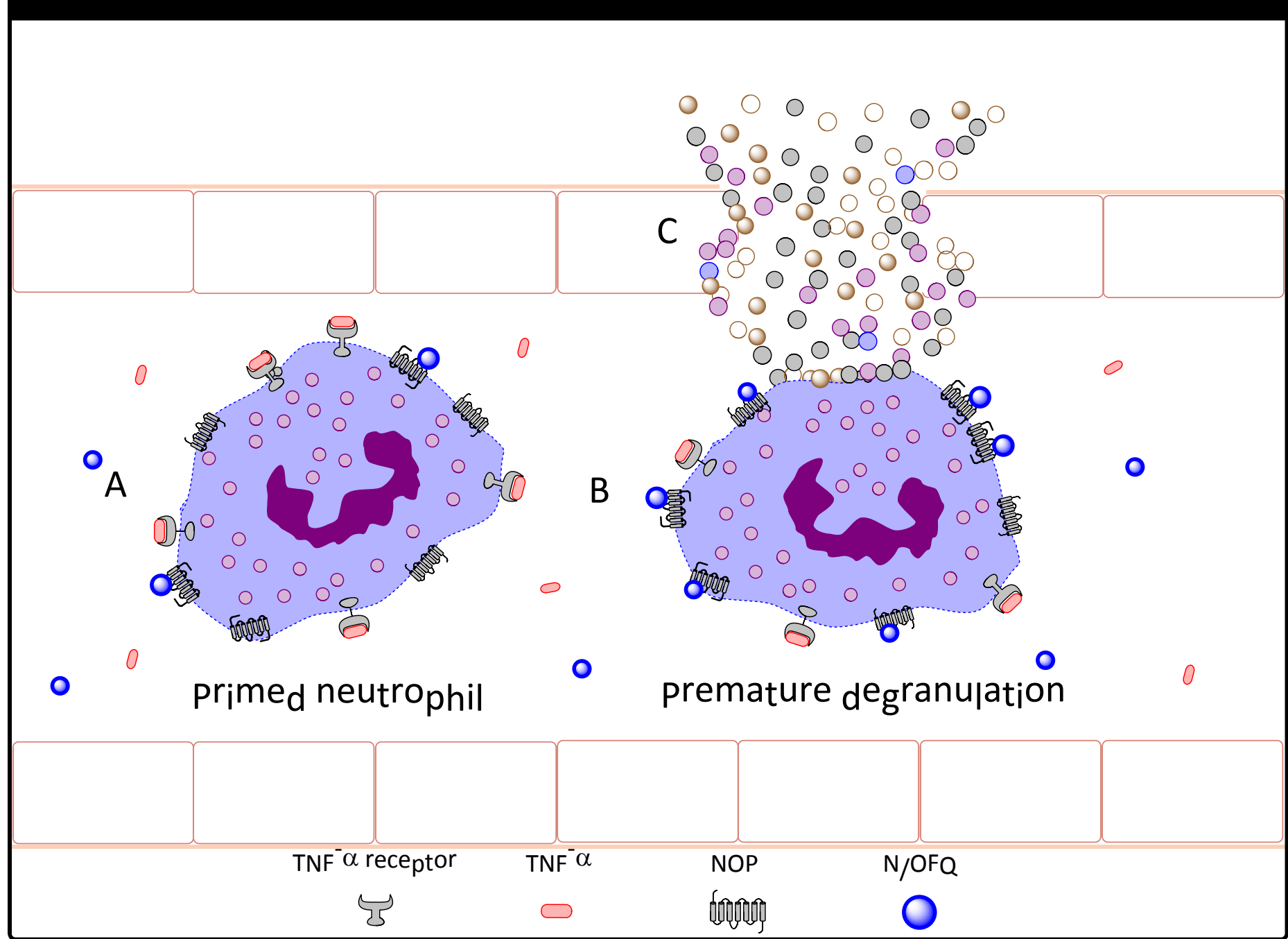

Figure 2 Hypothetical mechanism of N/OFQ-induced vascular permeability. The high concentration of TNF- $\alpha$ has delayed apoptosis of the neutrophil and prevented it from migrating to the tissue. N/OFQ in plasma has bound to NOP, leading to a primed state. b) Increased plasma N/OFQ concentrations result in further encounters which stimulates degranulation. c) Neutrophil products have damaged the vascular endothelium leading to increased permeability. 
Some of the Diagnostic criteria for Sepsisi3

- Leukocytosjs or LeukOpenja

- Hypotensjon

- Hypoxemia

- Decreased capillary refill or mottling

- Increased heart rate

- Significant edema

- Temperature $>38.3^{\circ} \mathrm{C}$ or $<36^{\circ} \mathrm{C}$

- coagulation abnormalities

- Hyperactatemia cellular changes during sepsis and the processes they may contribute to

- Increase $e_{\text {histamine rejease }} \rightarrow$ vasodilation $\rightarrow$ Increase $_{d}$ permeability of the

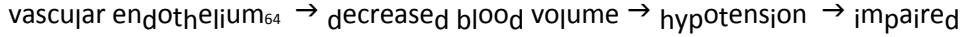
cardiac function $\rightarrow$ hypoxemia $\rightarrow$ organ dysfunction

- Upregulation of adhesion molecules $16 \cdot 17 \rightarrow$ increased leucocyte rolling and adhesjon $\rightarrow$ microvascuar occjusjon $\rightarrow$ hypoxemia $\rightarrow$ organ dysfunction.

- Increase deucocyte count ${ }_{13} \rightarrow{ }_{\text {increase }}$ rejease of lytic enzymes $\rightarrow$ damage

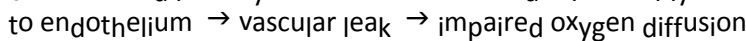

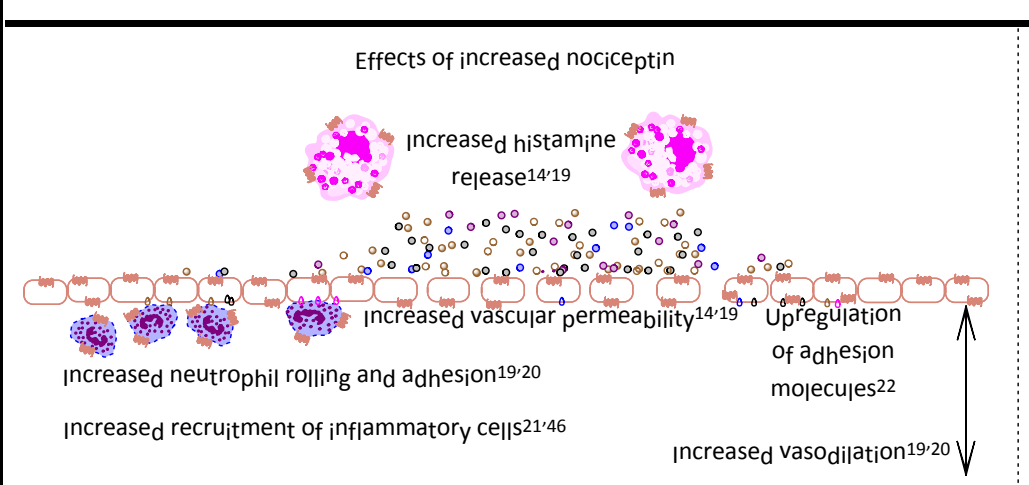

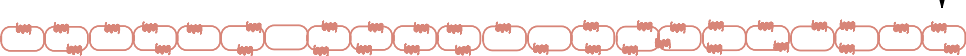

Decreased survival in septic rats 46 Decreased survival in patients with sepsis37
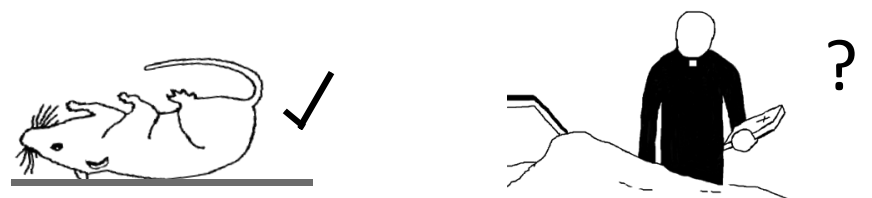

Effects of decrease $_{d}$ nociceptin

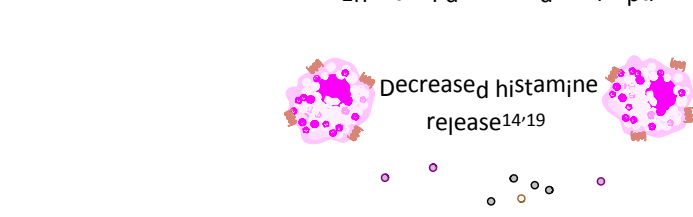

Reduced leucocyte rolling

Decreased recruitment

ment

$\left.\lim _{m \rightarrow \infty}\right)_{m}$ Decreased vasodilation $19 \cdot 20$

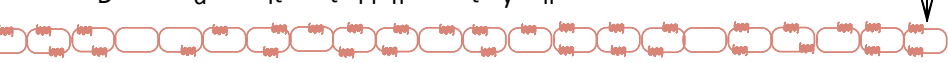

ncreased survival in septic rats

increased survival in patients with sepsis37

\section{iो}

Leucocyte expressing NOP

Endothelial ce|| expressjng NOP
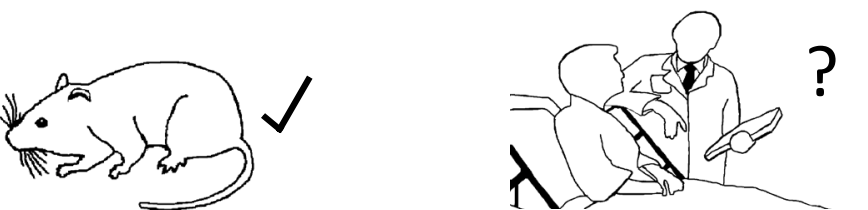

stis

Mast ce|| expressing NOP

Figure 3. Sepsis and the effects of increased and decreased nociceptin. Top: Some of the diagnostic criteria, changes and processes of sepsis. Middle: Effects of N/OFQ that may be related to sepsis. Bottom left: Effects of N/OFQ on survival in rats. Bottom right: Possible effects of N/OFQ on survival in humans. 


\section{References}

1. Sriskandan S, Altmann D. The immunology of sepsis. J Pathol. 2008;214:211-23

2. Jawad I, Lukšić I, Rafnsson SB. Assessing available information on the burden of sepsis: global estimates of incidence, prevalence and mortality. Journal of global health 2012; 2; 010404

3. Martin GS. Sepsis, severe sepsis and septic shock: changes in incidence, pathogens and outcomes. Expert review of anti-infective therapy 2012; 10: 701-6

4. Vincent J-L, Sakr Y, Sprung CL, et al. Sepsis in European intensive care units: Results of the SOAP study*. Critical Care Medicine 2006; 34: 344-53

5. Serrano-Gomez A, Thompson J, Lambert D. Nociceptin/orphanin FQ in inflammation and sepsis. British Journal of Anaesthesia 2011; 106: 6-12

6. Gavioli EC, Romao PR. NOP Receptor Ligands as Potential Agents for Inflammatory and Autoimmune Diseases. Journal of amino acids 2011; 2011: 836569

7. Reinscheid RK, Nothacker H-P, Bourson A, et al. Orphanin FQ: a neuropeptide that activates an opioidlike G protein-coupled receptor. Science 1995; 270: 792-4

8. Lambert DG. The nociceptin/orphanin FQ receptor: a target with broad therapeutic potential. Nature reviews Drug discovery 2008; 7: 694-710

9. Pampusch MS, Serie JR, Osinski MA, Seybold VS, Murtaugh MP, Brown DR. Expression of nociceptin/OFQ receptor and prepro-nociceptin/OFQ in lymphoid tissues. Peptides 2000; 21: 1865-70

10. Arjomand J, Cole S, Evans CJ. Novel orphanin $\mathrm{FQ} /$ nociceptin transcripts are expressed in human immune cells. Journal of neuroimmunology 2002; 130: 100-8

11. Miller TR, Fulford AJ. Regulation of nociceptin/orphaninFQ secretion by immune cells and functional modulation of interleukin-2. Peptides 2007; 28: 2243-52

12. Levy MM, Fink MP, Marshall JC, et al. $2001 \mathrm{sccm} / \mathrm{esicm} / \mathrm{accp} / \mathrm{ats} / \mathrm{sis}$ international sepsis definitions conference. Intensive care medicine 2003; 29: 530-8

13. Kimura T, Kitaichi K, Hiramatsu K, et al. Intradermal application of nociceptin increases vascular permeability in rats: the possible involvement of histamine release from mast cells. European journal of pharmacology 2000; 407: 327-32

14. Adib-Conquy M, Cavaillon J-M. Stress molecules in sepsis and systemic inflammatory response syndrome. FEBS Lett. 2007;581: 3723-33.

15. Jean-Baptiste E. Cellular mechanisms in sepsis. J Intensive Care Med. 2007;22:63-72.

16. Marshall JS, Jawdat DM. Mast cells in innate immunity. J Allergy Clin Immunol. 2004;114:217

17. Aird WC. The role of the endothelium in severe sepsis and multiple organ dysfunction syndrome. Blood 2003; 101: 3765-77

18. Brookes ZL, Stedman EN, Guerrini R, Lawton BK, Calo G, Lambert DG. Proinflammatory and vasodilator effects of nociceptin/orphanin $\mathrm{FQ}$ in the rat mesenteric microcirculation are mediated by histamine. American Journal of Physiology-Heart and Circulatory Physiology 2007; 293: H2977-H85

19. Calo G, Guerrini R, Rizzi A, Salvadori S, Burmeister M, Kapusta DR, Lambert DG, Regoli D. UFP-101, a peptide antagonist selective for the nociceptin/orphanin FQ receptor. CNS Drug Rev. 2005;11:97-112

20. Brookes ZL, Stedman EN, Brown NJ, et al. The Nociceptin/Orphanin FQ Receptor Antagonist UFP-101 Reduces Microvascular Inflammation to Lipopolysaccharide In Vivo. PloS one 2013; 8: e74943

21. Serhan CN, Fierro IM, Chiang N, Pouliot M. Cutting edge: nociceptin stimulates neutrophil chemotaxis and recruitment: inhibition by aspirin-triggered-15-epi-lipoxin A4. The Journal of Immunology 2001; 166: 3650-4 
22. Kato S, Tsuzuki Y, Hokari R, et al. Role of nociceptin/orphanin FQ (Noc/oFQ) in murine experimental colitis. Journal of neuroimmunology 2005; 161: 21-8

23. Fink M, Evans T. Mechanisms of organ dysfunction in critical illness: report from a Round Table Conference held in Brussels. Intensive care medicine 2002; 28: 369-75

24. Astiz ME, DeGent GE, Lin RY, Rackow EC. Microvascular function and rheologic changes in hyperdynamic sepsis. Critical Care Medicine 1995; 23: 265-71

25. Trombella S, Vergura R, Falzarano S, Guerrini R, Calo G, Spisani S. Nociceptin/orphanin FQ stimulates human monocyte chemotaxis via NOP receptor activation. Peptides 2005; 26: 1497-502

26. Brown KA, Brain SD, Pearson JD, Edgeworth JD, Lewis SM, Treacher DF. Neutrophils in development of multiple organ failure in sepsis. The Lancet 2006; 368: 157-69

27. Thompson JP, Serrano-Gomez A, McDonald J, Ladak N, Bowrey S, Lambert DG. The Nociceptin/Orphanin FQ System Is Modulated in Patients Admitted to ICU with Sepsis and after Cardiopulmonary Bypass. PloS one 2013; 8: e76682

28. Cohen J. The immunopathogenesis of sepsis. Nature 2002; 420: 885-91

29. Jimenez MF, Watson RWG, Parodo J, et al. Dysregulated expression of neutrophil apoptosis in the systemic inflammatory response syndrome. Archives of Surgery 1997; 132: 1263

30. Colotta F, Re F, Polentarutti N, Sozzani S, Mantovani A. Modulation of granulocyte survival and programmed cell death by cytokines and bacterial products. Blood 1992; 80: 2012-20

31. Otsuka $\mathrm{Y}$, Nagano $\mathrm{K}$, Hori K, et al. Inhibition of neutrophil migration by tumor necrosis factor. Ex vivo and in vivo studies in comparison with in vitro effect. The Journal of Immunology 1990; 145: 2639-43

32. Martins PS, Kallas EG, Neto MC, Dalboni MA, Blecher S, Salomao R. Upregulation of reactive oxygen species generation and phagocytosis, and increased apoptosis in human neutrophils during severe sepsis and septic shock. Shock 2003; 20: 208-12

33. Simms H, D'amico R. Polymorphonuclear leukocyte dysregulation during the systemic inflammatory response syndrome. Blood. 1994;83:1398-407

34. Nakamori Y, Koh T, Ogura H, et al. Enhanced Expression of Intranuclear NF-[kappa] B in Primed Polymorphonuclear Leukocytes in Systemic Inflammatory Response Syndrome Patients. The Journal of Trauma and Acute Care Surgery 2003; 54: 253-60

35. Williams JP, Thompson JP, Young SP, et al. Nociceptin and urotensin-II concentrations in critically ill patients with sepsis. Br J Anaesth 2008; 100: 810-4

36. Serhan $\mathrm{CN}$. Resolution phase of inflammation: novel endogenous anti-inflammatory and proresolving lipid mediators and pathways. Annu Rev Immunol 2007; 25: 101-37

37. Amano H, Morimoto K, Senba M, et al. Essential contribution of monocyte chemoattractant protein-1/CC chemokine ligand- 2 to resolution and repair processes in acute bacterial pneumonia. The Journal of Immunology 2004; 172: 398-409

38. Peluso J, LaForge KS, Matthes HW, Kreek MJ, Kieffer BL, Gavériaux-Ruff C. Distribution of nociceptin/orphanin FQ receptor transcript in human central nervous system and immune cells. Journal of neuroimmunology 1998; 81: 184-92

39. Strauss-Ayali D, Conrad SM, Mosser DM. Monocyte subpopulations and their differentiation patterns during infection. J Leukoc Biol. 2007;82:244-52.

40. Warren HS. Editorial: control issues in sepsis: what modulates apoptosis? J Leukoc Biol. 2011;89:325-6

41. Buzas B, Rosenberger J, Kim KW, Cox BM. Inflammatory mediators increase the expression of nociceptin/orphanin FQ in rat astrocytes in culture. Glia 2002; 39: 237-46

42. Dinarello CA. Immunological and inflammatory functions of the interleukin-1 family. Annual review of immunology 2009; 27: 519-50

43. Zhao H, Wu G-C, Cao X-D. Immunomodulatory activity of orphanin FQ/nociceptin on traumatic rats. Acta Pharmacologica Sinica 2002; 23: 343 
44. Goldfarb Y, Reinscheid RK, Kusnecov AW. Orphanin FQ/nociceptin interactions with the immune system in vivo: gene expression changes in lymphoid organs and regulation of the cytokine response to staphylococcal enterotoxin A. Journal of neuroimmunology 2006; 176 : 76-85

45. Carvalho D, Petronilho F, Vuolo F, et al. The nociceptin/orphanin FQ-NOP receptor antagonist effects on an animal model of sepsis. Intensive care medicine 2008; 34: 2284-90

46. Kaminsky DE, Rogers TJ. Suppression of CCL2/MCP-1 and CCL5/RANTES expression by nociceptin in human monocytes. Journal of Neurolmmune Pharmacology 2008; 3: 75-82

47. Al-Hashimi M, Scott S, Thompson J, Lambert D. Opioids and immune modulation: more questions than answers. British Journal of Anaesthesia 2013; 111: 80-8

48. Hotchkiss RS, Karl IE. The pathophysiology and treatment of sepsis. New England Journal of Medicine 2003; 348: 138-50

49. Németh J, Helyes Z, Oroszi G, Thán M, Pintér E, Szolcsányi J. Inhibition of nociceptin on sensory neuropeptide release and mast cell-mediated plasma extravasation in rats. European journal of pharmacology 1998; 347: 101-4

50. Helyes Z, Németh J, Pintér E, Szolcsányi J. Inhibition by nociceptin of neurogenic inflammation and the release of SP and CGRP from sensory nerve terminals. British journal of pharmacology 1997; 121: 613-5

51. Petrella C, Giuli C, Broccardo M, et al. Protective and worsening peripheral nociceptin/orphanin FQ receptor-mediated effect in a rat model of experimental colitis. Pharmacological research : the official journal of the Italian Pharmacological Society 2013; 70: 72-9

52. Kapusta DR. Neurohumoral effects of orphanin FQ/nociceptin: relevance to cardiovascular and renal function. Peptides 2000; 21: 1081-99

53. Hashiba E, Hirota K, Kudo T, Guerrini R, Matsuki A. Effects of nociceptin/orphanin FQ receptor ligands on blood pressure, heart rate, and plasma catecholamine concentrations in guinea pigs. Naunyn-Schmiedeberg's archives of pharmacology 2003; 367: 342-7

54. Madeddu P, Salis MB, Milia AF, et al. Cardiovascular effects of nociceptin in unanesthetized mice. Hypertension 1999; 33: 914-9

55. Neal Jr CR, Akil H, Watson Jr SJ. Expression of orphanin FQ and the opioid receptor-like (ORL1) receptor in the developing human and rat brain. Journal of chemical neuroanatomy 2002; 22: 219-49

56. Burmeister MA, Kapusta DR. Centrally administered nociceptin/orphanin FQ (N/OFQ) evokes bradycardia, hypotension, and diuresis in mice via activation of central N/OFQ peptide receptors. Journal of Pharmacology and Experimental Therapeutics 2007; 322: 324-31

57. Burmeister MA, Ansonoff MA, Pintar JE, Kapusta DR. Nociceptin/orphanin FQ (N/OFQ)evoked bradycardia, hypotension, and diuresis are absent in N/OFQ peptide (NOP) receptor knockout mice. Journal of Pharmacology and Experimental Therapeutics 2008; 326: 897-904

58. Chu X, Xu N, Li P, Mao L, Wang JQ. Inhibition of cardiovascular activity following microinjection of novel opioid-like neuropeptide nociceptin (orphanin FQ) into the rat rostral ventrolateral medulla. Brain research 1999; 829: 134-42

59. Laufenberg $\mathrm{L}$, Weller $\mathrm{GE}$, Lang $\mathrm{CH}$, Ruiz-Velasco V. Nociceptin receptor signaling in sympathetic neurons from septic rats. Journal of Surgical Research 2013

60. Stamer UM, Book M, Comos C, Zhang L, Nauck F, Stuber F. Expression of the nociceptin precursor and nociceptin receptor is modulated in cancer and septic patients. BJA: British Journal of Anaesthesia 2011; 106: 566-72

61. Zhang L, Stuber F, Stamer UM. Inflammatory mediators influence the expression of ociceptin and its receptor in human whole blood cultures. PLoS One. 2013;8:e74138. 